\title{
Bmi1 knockdown inhibits hepatocarcinogenesis
}

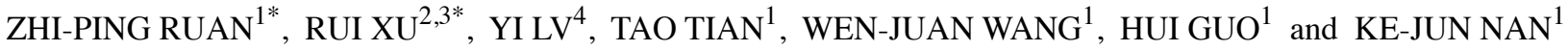 \\ ${ }^{1}$ Department of Oncology, First Affiliated Hospital, Medical College of Xi'an Jiaotong University; \\ ${ }^{2}$ Medical College of Xi'an Jiaotong University; ${ }^{3}$ First Department of Internal Medicine, \\ Shaanxi Cancer Hospital; ${ }^{4}$ Department of Hepatobiliary Surgery, First Affiliated Hospital, \\ Medical College of Xi'an Jiaotong University, Xi'an, Shaanxi, P.R. China
}

Received August 27, 2012; Accepted October 12, 2012

DOI: $10.3892 /$ ijo.2012.1693

\begin{abstract}
Although Bmil is well established as one of the most commonly activated oncogenes, the precise role of Bmil during hepatocarcinogenesis remains unclear. In addition, Bmil provides a potential therapeutic target for the future treatment of hepatocellular carcinoma (HCC). In this study, the expression of Bmil in HCC tissues was evaluated by immunohistochemistry and western blot analysis. We found that Bmil was much more highly expressed in HCC tissue compared to normal liver tissue. The shRNA-mediated knockdown of Bmil was used to assess the effects of Bmil in hepatocarcinogenesis. Bmil downregulation reduced cell growth and tumorsphere formation in vitro. A cell cycle analysis using flow cytometry clarified that Bmil knockdown blocked the cell cycle transition from the G0/G1 to the S phase. Additionally, the Bmil knockdown led to reduced tumorigenicity in vivo. Furthermore, Bmil expression enhanced the sensitivity of $\mathrm{HCC}$ to the therapeutic agent, sorafenib. Taken together, the current results demonstrate that Bmil functions as a promoter in cell proliferation and hepatocarcinogenesis, providing a potential therapeutic target for the future treatment of HCC.
\end{abstract}

\section{Introduction}

Hepatocellular carcinoma (HCC) is the third leading cause of mortality worldwide; there are 600,000 estimated new HCC cases annually and almost as many as deaths (1). This malignancy occurs more often in men than in women, with higher incidence rates reported in several areas of Asia and Africa.

Sorafenib is one of the FDA-approved molecular targeted drugs for advanced HCC, and it confers significantly improved survival. Despite such advances in HCC therapy, the poor prog-

Correspondence to: Professor Ke-Jun Nan, Department of Oncology, First Affiliated Hospital, Medical College of Xi'an Jiaotong University, 277 Yantaxi Road, Xi'an, Shaanxi 710061, P.R. China

E-mail: nankejun@126.com

${ }^{*}$ Contributed equally

Key words: hepatocellular carcinoma, Bmil, proliferation, sorafenib nosis of HCC is still unavoidable due to the rapidly dividing cells that are the primary targets of traditional anticancer therapy (2). In the cancer stem cell (CSC) theory, only a limited number of cells within the tumor, which are termed CSCs, are proposed to persist in tumors as a distinct population and cause relapse and metastasis by giving rise to new tumors. CSCs have been identified and isolated from hematopoietic malignancies and other solid tumors, including glioblastoma, breast cancer, colon cancer and hepatocellular carcinoma (3-7). Currently, there is no drug that specifically targets this fraction of tumor cells; therefore, CSC-targeted anticancer interventions are potential therapies for this malignancy.

The epigenetic regulator polycomb group (PcG) genes are thought to control cell fate, cell differentiation and cancer development. Bmil is one of the core components of the $\mathrm{PcG}$ protein complex, which is involved in axial patterning, hematopoiesis, cell proliferation and senescence (8-11). Bmil was first identified as an oncogene for the generation of B- or T-cell leukemia in cooperation with c-Myc, which is dysregulated in various human cancers, such as colorectal carcinoma, HCC and lung cancer (12-14). Furthermore, Bmil as a stem cell gene has been defined by the fact that its deficiency leads to compromised adult stem cell function (15). It has been demonstrated that Bmil is necessary for the maintenance of stemness in leukemic stem cells and solid tumor stem cells, including HCC cells (16-18). Importantly, the overexpression of Bmil correlates with therapy failure in many tumor types, including those in breast, prostate, lung and ovarian cancer patients $(14,19,20)$.

In the present study, we performed detailed analyses to examine the roles of Bmil in HCC. Bmil expression was evaluated by western blot analysis and immunohistochemical staining in normal liver and HCC tissues. Bmil knockdown in the HCC cell lines inhibited tumorsphere formation in vitro and cell growth and tumor formation in vivo. A cell cycle analysis clarified that the knockdown of Bmil induced cell cycle arrest. Furthermore, Bmil knockdown also enhanced the sensitivity of HCC to the therapeutic agent, sorafenib.

\section{Materials and methods}

Patients and clinicopathological analysis. Surgical resection samples were obtained from 47 patients (including 9 females and 38 males) diagnosed with HCC at the First Affiliated 
Hospital, Medical College of Xi'an Jiaotong University, Xi'an, China from 2001 to 2003. The clinicopathological data of these patients, including the tumor stage, grade, differentiation, and survival time, were collected, and the follow-up data were updated through June 2006. A total of 9 match-paired HCC tissues and adjacent non-tumor tissues were collected from the Department of Hepatobiliary Surgery, First Affiliated Hospital, Medical College of Xi'an Jiaotong University. All of the tissue samples were obtained from untreated patients who were undergoing surgery. The study was approved by the Medical Ethics Committee of the First Affiliated Hospital, Medical College of Xi'an Jiaotong University, and all the patients formally consented to be a part of the study to the best of their understanding.

Immunohistochemistry. A standard immunostaining procedure was performed using mouse monoclonal antibodies against Bmil (Millipore; Boston, MA, USA), proliferating cell nuclear antigen (PCNA; Maixin Bio, Fujian, China), Ki-67 (Maixin Bio) or an isotype-matched control antibody. The immunoreactivity and subcellular localization of Bmil were evaluated independently by 3 investigators.

Bmil staining was classified into 2 groups, negative or positive, based on the percentage of positive cells and the staining intensity. The percentage of positive cells was divided into 4 ranks of scores: $<10 \%$ (1), $10-25 \%$ (2), $25-50 \%$ (3) and $>50 \%$ (4). The intensity of staining was also divided into 4 ranks of scores: no staining (1), light brown (2), brown (3) and dark brown (4). The positivity of Bmil staining was determined by the following formula: immunohistochemistry score $=$ percentage score $\mathrm{x}$ intensity score. An overall score of $\leq 8$ was defined as negative and $>8$ as positive.

Cells and cell culture. The HCC-derived cell lines, Bel-7402 (CCTCC GDC035), SMMC-7721 (CCTCC GDC064) and HepG2 (CCTCC GDC024), were all purchased from the China Center for Type Culture Collection (CCTCC; Wuhan, China). The cells were maintained in RPMI-1640 medium (Invitrogen, Carlsbad, CA, USA) supplemented with 10\% fetal bovine serum (FBS, Invitrogen) in a humidified atmosphere at $37^{\circ} \mathrm{C}$ with $5 \% \mathrm{CO}_{2}$. The MHCC97 cells (Cell Bank of Chung Shan Hospital, Shanghai, China) were maintained in Dulbecco's modified Eagle's medium (DMEM, Invitrogen) supplemented with $10 \%$ FBS in a humidified atmosphere at $37^{\circ} \mathrm{C}$ with $5 \% \mathrm{CO}_{2}$.

Bmil shRNA vector construction and transfection. The oligonucleotide insert for the hairpin siRNA targeting the Bmil mRNA sequences was GGAGGAACCTTTAAAGGA TTA. The oligonucleotide sequences were 5'-CACCGGAG GAACCTTTAAAGGATTATTCAAGAGATAATCCTTTAA AGGTTCCTCCTTTTTTG-3' and 5'-GATCCAAAAAAGG AGGAACCTTTAAAGGATTATCTCTTGAATAATCCTTT AAAGGTTCCTCC-3'. The synthesized oligonucleotide (GenePharma, Shanghai, China) inserts were annealed and cloned into the PGPU6/GFP/neo-shRNA expression vector (GenePharma) to generate PGPU6/GFP/neo-shBmil. The plasmid PGPU6/GFP/neo-shControl (GenePharma) was used as the negative control and encoded a hairpin siRNA with a nonsense sequence.
For stable cell line generation, the transfection was performed using Lipofectamine 2000 (Invitrogen) following the manufacturer's instructions. Bmil stable-knockdown cells and the control cells were selected using $0.8 \mathrm{mg} / \mathrm{ml} \mathrm{G} 418$ (Calbiochem, La Jolla, CA, USA).

Western blot analysis. Western blot analyses were performed as previously described using cell lysates (21). The crude proteins were then subjected to SDS-PAGE and then transferred onto a PVDF membrane. After blocking, the membrane was incubated with the appropriate antibody against Bmi1 (Millipore) or $\beta$-actin (Santa Cruz Biotechnology, Santa Cruz, CA, USA) at $4^{\circ} \mathrm{C}$ overnight, followed by a horseradish peroxidase-labeled secondary antibody. The blots were developed using a chemiluminescent detection system (Amersham Life Science, Buckinghamshire, UK).

Cell cycle assay. Cells $\left(1 \times 10^{6}\right)$ were cultured in 6-well plates for $24 \mathrm{~h}$ and then harvested and washed with PBS, followed by fixation with $70 \%$ ethanol overnight at $4{ }^{\circ} \mathrm{C}$. After washing with PBS twice, the cells were stained in PBS with $50 \mu \mathrm{g} / \mathrm{ml}$ propidium iodide (PI; Sigma, St. Louis, MO, USA) and $10 \mu \mathrm{g} / \mathrm{ml}$ RNase A (Sigma) at room temperature in the dark. The cell cycle was assessed by flow cytometry (FACSCalibur; BD Biosciences, Franklin Lakes, NJ, USA), and the data were analyzed with the FACSCalibur flow cytometer using ModFit LT software.

Cell proliferation and colony formation assay. Cell proliferation was evaluated on days $1,3,5$, and 7 after seeding the cells $\left(5 \times 10^{4}\right)$ in triplicate in 6 -well plates. A total of 200 cells plated on 100-mm cell culture dishes in triplicate were cultured for 3 weeks. The cell colonies were stained with Giemsa solution after being fixed in methanol for $15 \mathrm{~min}$ at room temperature.

Tumor xenografts. The stable Bmi1 knockdown or control cell line $\left(10^{6}\right.$ cells) was injected into the subcutaneous tissue in the dorsum of 4-6-week-old male Balb/c-nude mice. Three animals per group were used in each experiment. The tumors were measured weekly using a vernier caliper, and the volume was calculated according to the following formula: length $\mathrm{x}$ width $2 / 2$. At the end of the experiment, the tumors were dissected, and their net weights were measured. The experimental protocols were evaluated and approved by the Animal Care and Use Committee of the Medical College of Xi'an Jiaotong University.

Drug experiments. Sorafenib was dissolved in DMSO (Sigma) and diluted with RPMI-1640 to the desired concentration $(5 \mu \mathrm{M})$, with a final DMSO concentration of $0.1 \%$ for the in vitro studies. DMSO at $0.1 \%(\mathrm{v} / \mathrm{v})$ was used as a solvent control.

Cells $\left(1 \times 10^{3}\right)$ were plated on $100-\mathrm{mm}$ cell culture dishes in triplicate, and cultured for 3 weeks. Sorafenib or the solvent was added at the appropriate concentration after $24 \mathrm{~h}$. The cell colonies were stained with Giemsa solution after being fixed in methanol for $15 \mathrm{~min}$ at room temperature.

A total of 5,000 cells were inoculated in 96-well microtiter plates and incubated overnight at $37^{\circ} \mathrm{C}$ in a humidified incubator with $5 \% \mathrm{CO}_{2}$. The cell viability was quantified every day using 3-(4,5-dimethylthiazol-2-yl)-2,5-diphenyl tetrazolium 
A
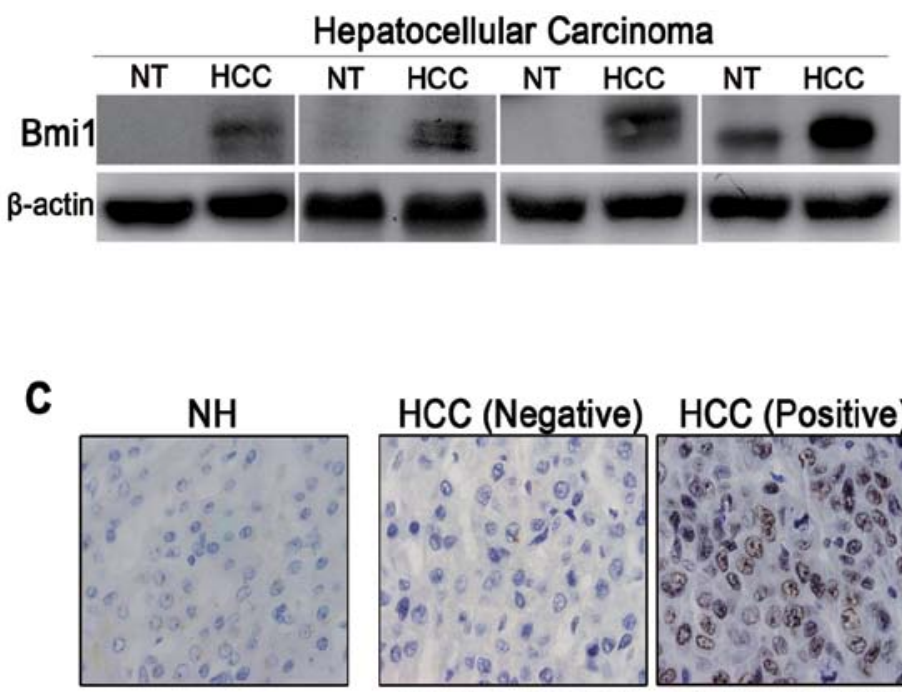

$(0 / 10,0 \%)$

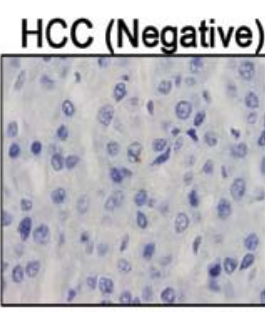

(30/47, 63.83\%)

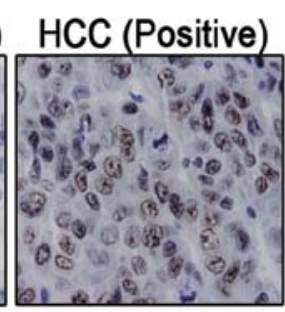

$(17 / 47,36.17 \%)$
B

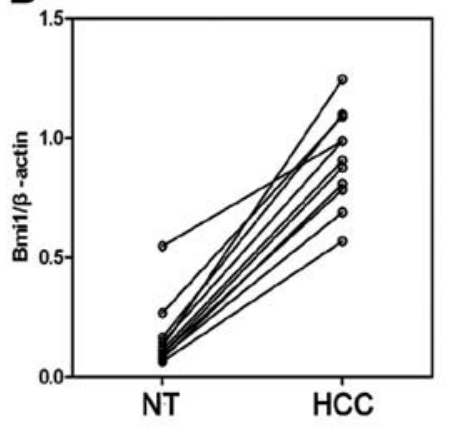

D

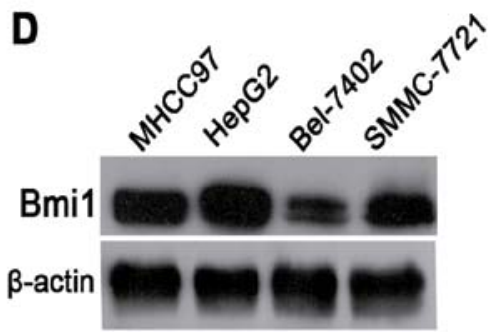

Figure 1. Bmil expression in human hepatocellular carcinoma (HCC) tissues and cell lines. (A) Representative western blots showing Bmil expression in 9 pairs of matched human HCC tissues and adjacent non-tumor (NT) tissues. (B) Bmil expression was compared between the matched, adjacent NT and HCC tissues in 9 patients $(\mathrm{P}<0.05)$. (C) Immunohistochemical staining is shown for Bmil in the HCC tissues and normal hepatic (NH) tissues. (D) Representative western blots showing Bmil expression in the HCC cell lines.

bromide (MTT; Sigma) staining, according to a standard protocol. The cells were incubated with sorafenib at various concentrations for an additional $72 \mathrm{~h}$ to evaluate the inhibitory effect of sorafenib on cell proliferation. The number of viable cells was determined by measuring the absorbance at $490 \mathrm{~nm}$.

Statistical analysis. All the experiments were repeated at least in triplicate. The data from all of the experiments were pooled, and the results are expressed as the means \pm SD. The statistical analysis was performed using SPSS 16.0 software (SPSS Inc.; Chicago, IL, USA). The two-tailed $\chi^{2}$ test was used to determine the significance of the differences between the co-variates. For 2-group analyses, Student's t-test was used to determine the statistical significance, whereas Pearson's linear regression analysis was performed to examine the correlation between 2 quantitative variables. $\mathrm{P}<0.05$ was considered to indicate a statistically significant difference.

\section{Results}

Bmil expression in human HCC tissues and cell lines. To evaluate Bmil expression, western blot analysis was performed using 9 pairs of HCC tissues and their corresponding adjacent non-tumor tissues. Bmil expression was normalized to $\beta$-actin expression for the semi-quantification analyses. As shown in Fig. 1A and B, Bmil expression was significantly higher in all 9 of the cancer tissues than in the matched nontumor tissues $(\mathrm{P}<0.001)$. Furthermore, to determine whether Bmil overexpression was linked to the clinical progression of HCC, 47 HCC tissues and 10 normal hepatic tissues were characterized for Bmil expression by immunohistochemistry. However, none of the 10 normal tissues were found to be
Table I. Summary of Bmi1 immunohistochemistry staining in hepatocellular carcinoma.

\section{Bmi1}

Characteristics

No. Negative Positive P-value

\begin{tabular}{lrrrr}
\hline $\begin{array}{l}\text { Age/year } \\
\leq 45\end{array}$ & 18 & 10 & 8 & 0.352 \\
$>45$ & 29 & 20 & 9 & \\
Histological grade & & & & \\
$\quad$ Well-moderate & 39 & 26 & 13 & 0.371 \\
$\quad$ Poor & 8 & 4 & 4 & \\
Extrahepatic metastasis & & & & \\
$\quad$ Absence & 15 & 7 & 8 & 0.094 \\
$\quad$ Present & 32 & 23 & 9 & \\
Tumor size & & & & \\
$\quad 550$ mm & 19 & 14 & 5 & 0.247 \\
$\quad 50$ mm & 28 & 16 & 12 & \\
Gender & & & & \\
$\quad$ Male & 38 & 26 & 12 & 0.178 \\
Female & 9 & 4 & 5 & \\
\hline
\end{tabular}

positive for Bmil, whereas Bmil expression was detected in 17 of the 47 cases of HCC (36.17\%) (Fig. 1C). In addition, there was no significant correlation between Bmil expression and clinicopathological features (Table I). Bmil expression in 4 HCC cell lines was detected using western blot analysis (Fig. 1D). Bmil showed a high level of expression in the HepG2 and MHCC97 cell lines. Taken together, the western 
A

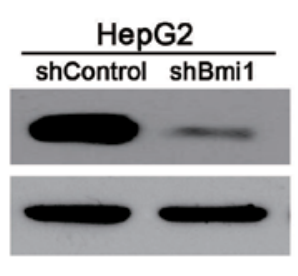

\section{C}

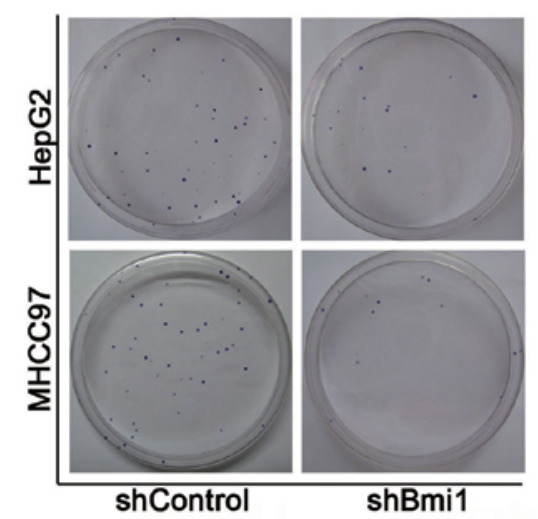

E

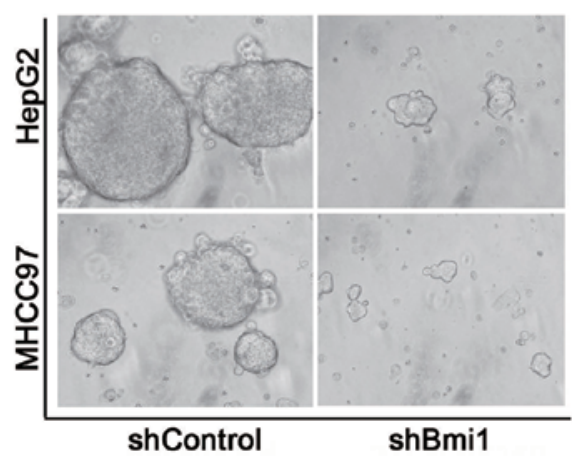

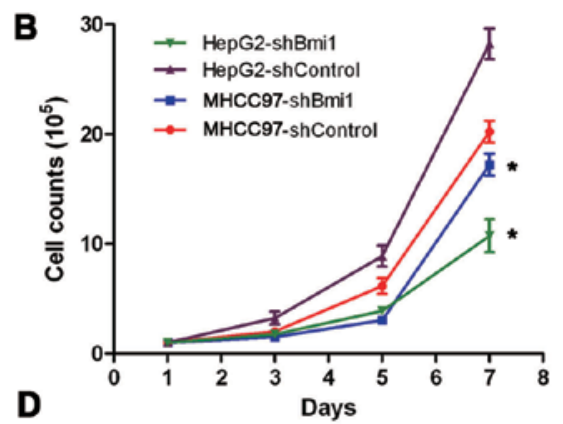

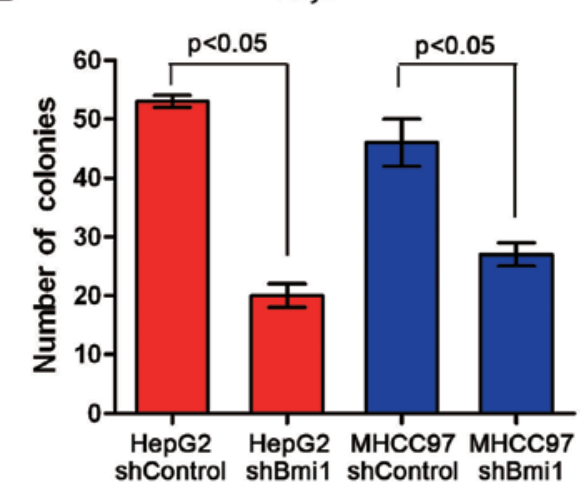

$\mathbf{F}$

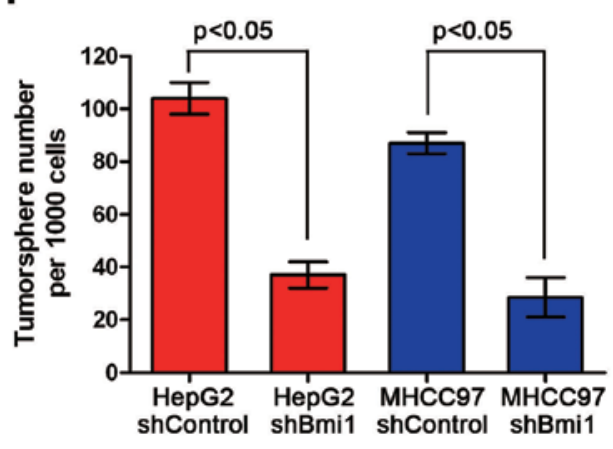

Figure 2. Bmil regulates the proliferation and tumorsphere formation of hepatocellular carcinoma cells. (A) Western blot assays were used to characterize the expression of shBmil in HepG2 and MHCC97 cells. (B) The proliferation of the HepG2-shControl cells (purple line), HepG2-shBmil cells (green line), MHCC97-shControl cells (red line) and MHCC97-shBmil (blue line) was determined by counting the cells longitudinally. (C) Clone formation assays of the shBmi1- and control vector-transfected HepG2 cells and MHCC97 cells. (D). Quantitative analysis of the clone formation assays. (E) Photomicrographs show the formed tumorspheres. (F) The charts illustrate the quantitative analysis of the tumorspheres.

blot and IHC semi-quantitative analyses consistently support the notion that Bmil upregulation is required for hepatocellular carcinogenesis, indicating that Bmil may function as an oncogene in HCC.

Bmil knockdown inhibits the proliferation and tumorsphere formation of HCC cells. To explore the role of Bmil in the development of HCC, we introduced shRNAs to suppress Bmil gene expression in HepG2 and MHCC97 cells (Fig. 2A); we also wished to examine how Bmil modulates the proliferation of HCC cells. The HepG2 and MHCC97 cells in which Bmil was knocked down (HepG2-shBmil and MHCC97-shBmi1) presented significantly lower proliferation rates than the HepG2-shControl and MHCC97-shControl cells. These results showed that the knockdown of Bmi1 expression significantly inhibited the in vitro proliferation of HCC cells (Fig. 2B). The clone formation assay showed that the Bmil knockdown cells formed fewer clones on the plates than the controls $(\mathrm{P}<0.05)$ (Fig. $2 \mathrm{C}$ and $\mathrm{D})$, suggesting that the knockdown of Bmil expression significantly inhibited the in vitro proliferation of HCC cells. Recently, a number of studies have indicated the existence of CSCs in HCC, cells that are critical for the maintenance of tumor growth, progression and metastasis $(22,23)$. It has been demonstrated that Bmil regulates the proliferation and differentiation of CSCs in other types of cancer in addition to HCC stem cell formation. We used a tumorsphere culture system to investigate the potential role of Bmil in tumorsphere formation. Fig. 2E illustrates that the knockdown of Bmil expression inhibited tumorsphere formation and growth, as evidenced by the significantly reduced numbers of tumorspheres compared with the numbers in the control cells $(\mathrm{P}<0.05)$. Our findings indicate that $\mathrm{Bmil}$ is a critical regulator of cell proliferation in $\mathrm{HCC}$ and tumorsphere-forming CSCs. 
A

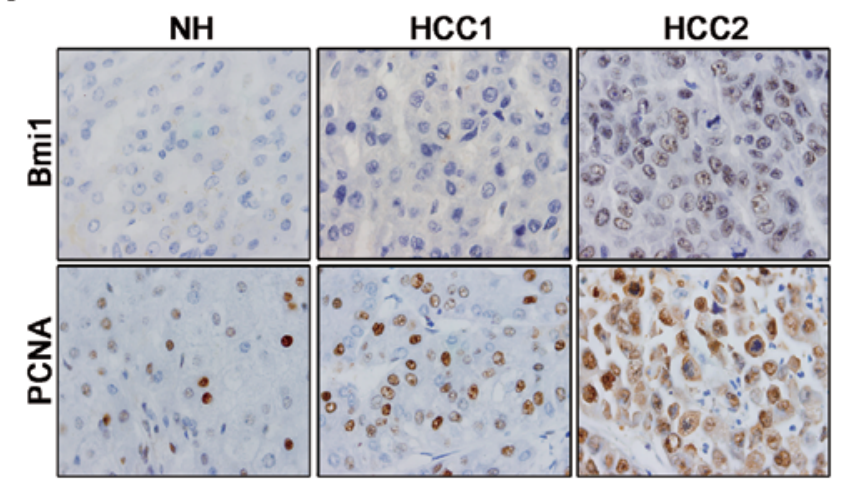

B

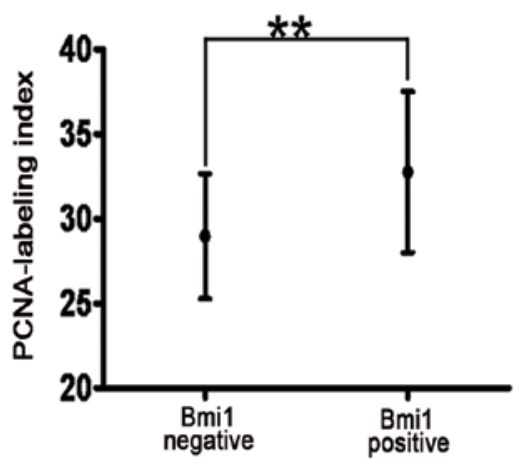

C

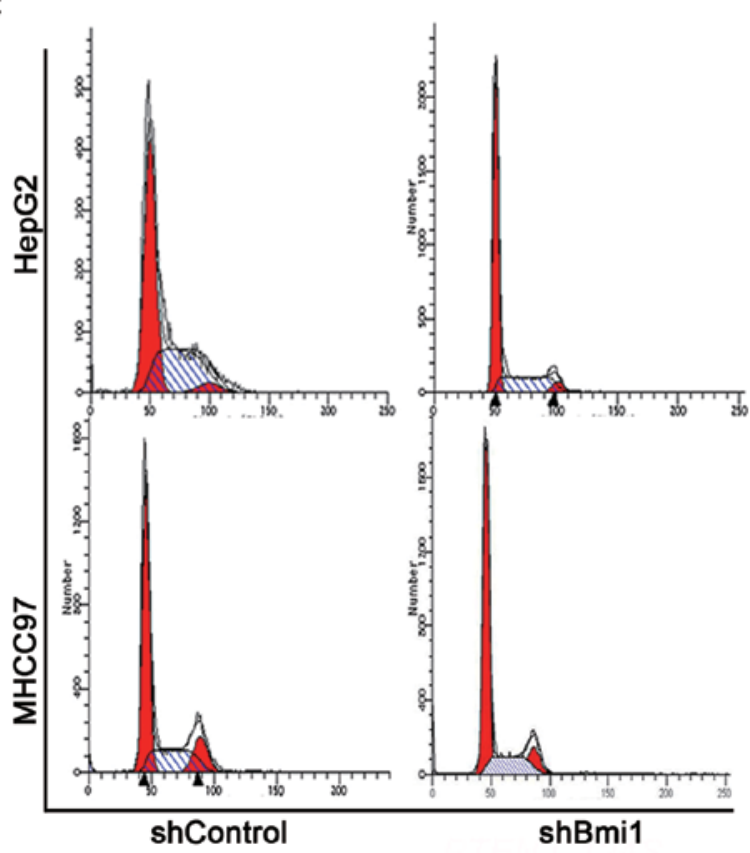

D

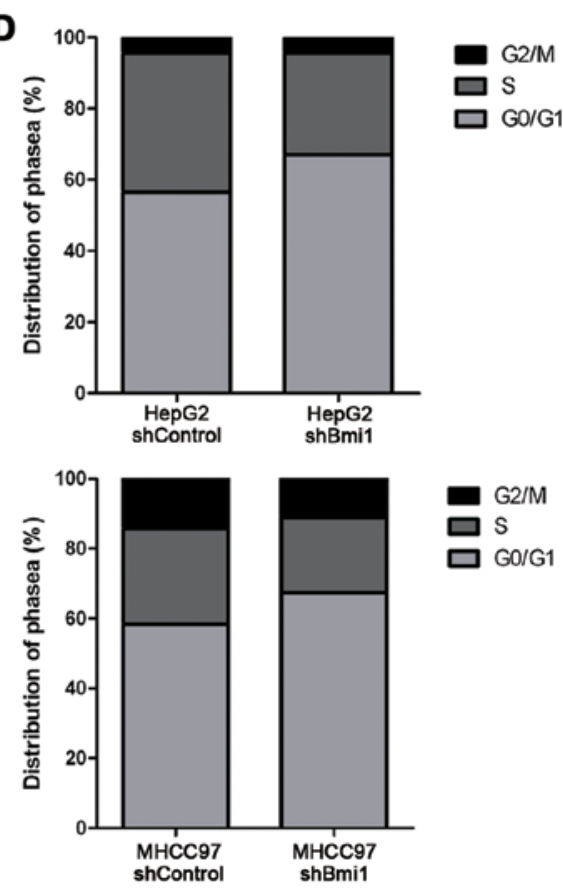

Figure 3. Bmil regulates the cell cycle of hepatocellular carcinoma (HCC) cells in vitro. (A) Immunohistochemical staining of Bmil and PCNA in normal hepatic (NH) tissue and HCC. (B) A semi-quantitative analysis of cell proliferation activity to determine the PCNA-labeling index (LI) in HCC. The results are shown as scatter plots of 5 randomly selected foci with or without Bmil or PTEN expression in HCC. ${ }^{* *} \mathrm{P}<0.01$. (C) Cell cycling analysis of the HepG2shControl cells, HepG2-shBmil cells, MHCC97-shControl cells and MHCC97-shBMI1 cells using flow cytometry. (D) A quantitative analysis is shown for the cell cycle distribution.

Bmil knockdown blocks the cell cycle transition from the G0/ G1 to the S phase. To determine whether Bmil is involved in the abnormal proliferation of $\mathrm{HCC}$, we examined PCNA staining in HCC pathological specimens. The PCNA labeling index (PCNA-LI) was the cell proliferation index, and we examined the correlation between Bmil staining and cell proliferation activity in HCC tissues. The results showed increased cell proliferation activity in the tissues with higher Bmil expression $(32.76 \pm 4.75$ vs. $28.97 \pm 3.71 \%$, Fig. $1 \mathrm{~A}$ and B). Changes in cell proliferation are typically associated with cell cycle modulation, and Bmil has been reported to promote the proliferation of a cervical cancer cell line by accelerating the cell cycle. To investigate the mechanisms by which Bmil regulates $\mathrm{HCC}$ cell proliferation, the cell cycle was investigated using flow cytometry after Bmil knockdown using shRNA. As shown in Fig. 3C, the percentage of HepG2-shControl cells in the G0/G1 phase was significantly greater $(71.52 \%)$ than that of HepG2-
shBmil cells (67.34\%). A similar trend was observed for the MHCC97-shControl cells (66.95\%) and MHCC97-shBmi1 cells $(56.63 \%)$, suggesting that the Bmil knockdown led to cell cycle arrest (Fig. 3D). Furthermore, there were no significant differences in cell apoptosis between the HepG2-shBmi1- and HepG2-control-transfected cells (data not shown), indicating that silencing Bmil inhibited tumorigenicity and was a result of the arrested cell cycle transition and not cell apoptosis.

Bmil knockdown inhibits the tumor formation of HCC cells in vivo. To validate the role of Bmil in tumor formation in vivo further, xenograft assays were performed by injecting Bmilsilenced HCC cells and control cells into nude mice. Although the HepG2-shControl and HepG2-shBmil cells induced palpable tumors by 8 weeks after the injection, the HepG2shBmil cells developed smaller tumors, with an average size of $678 \mathrm{~mm}^{3}$ and an average net weight of $0.77 \mathrm{~g}$, than those 
A

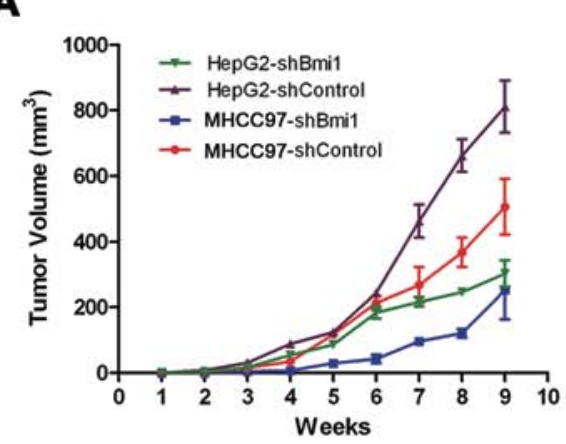

B

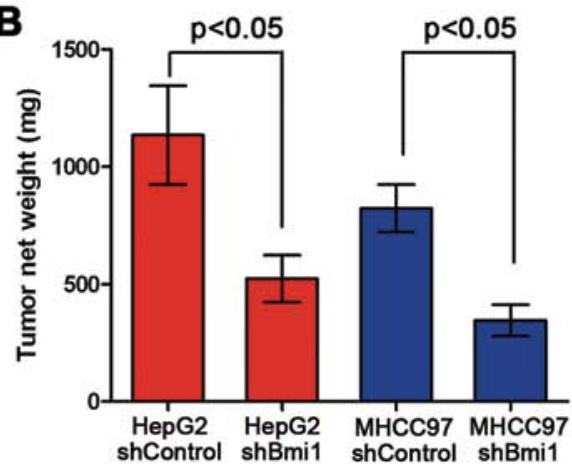

C

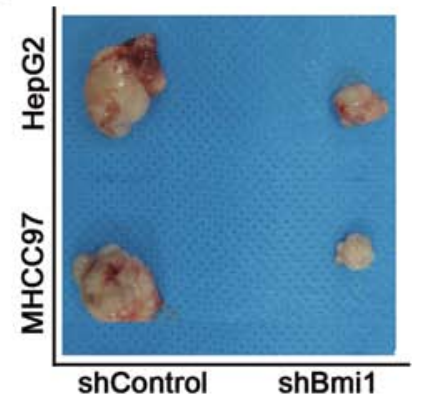

D

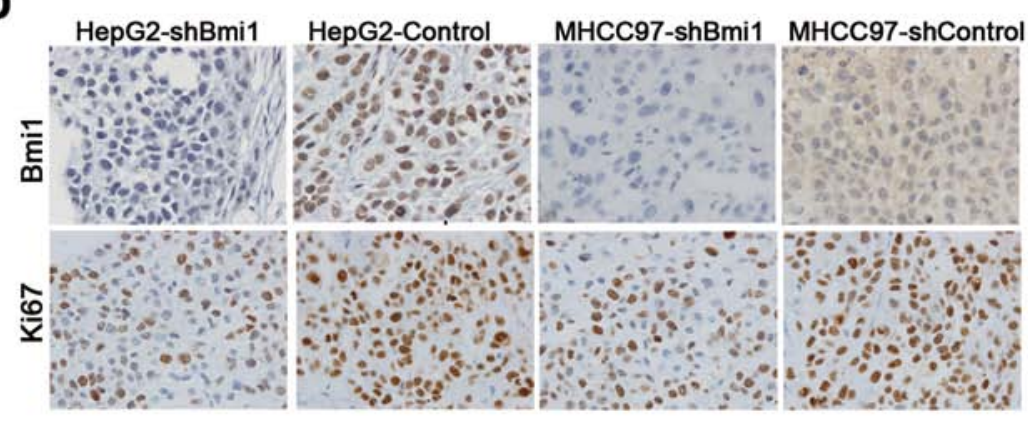

E

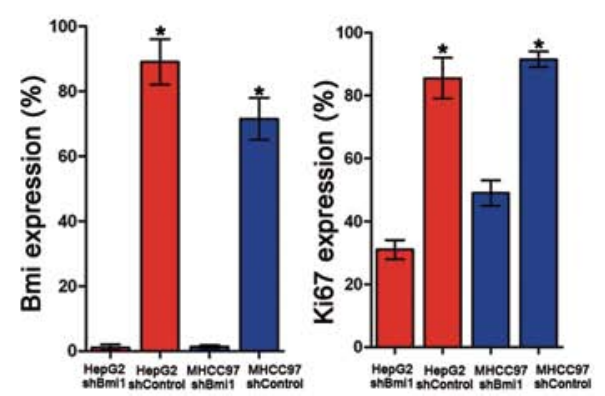

Figure 4. Bmil regulates the growth of hepatocellular carcinoma cells in vivo. (A) Tumor growth curves of the HepG2-shControl cells (purple line), HepG2shBmil cells (green line), MHCC97-shControl cells (red line) and MHCC97-shBmil (blue line) in Balb/c nude mice. At the end of the experiments, (B and C) the mice were sacrificed, the tumors were dissected, and the tumor net weights were measured. The data are shown as the means \pm standard error for each group ( $=6$ ); ${ }^{*} \mathrm{P}<0.05$. (D) Immunochemical staining for Ki67 and Bmil in the tumor xenografts. (E) A semi-quantitative analysis of Ki67 and Bmil in the tumor xenografts.

A

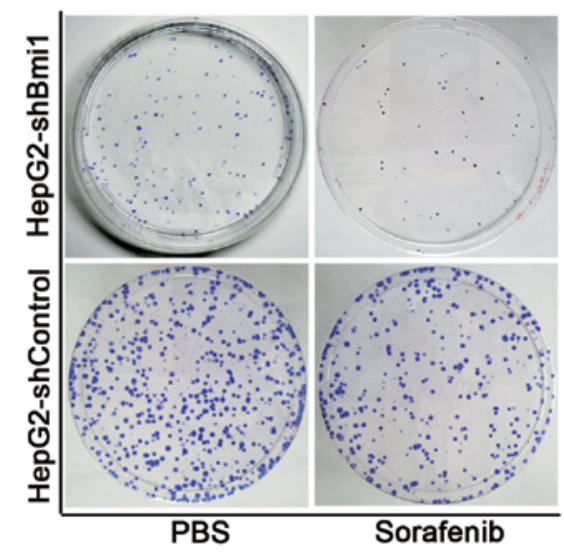

C

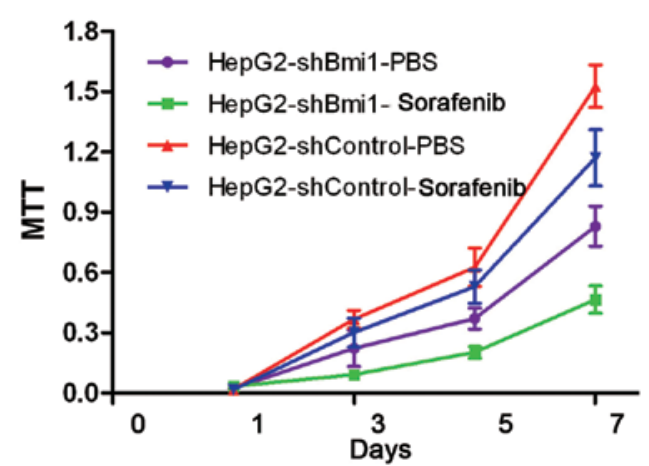

B

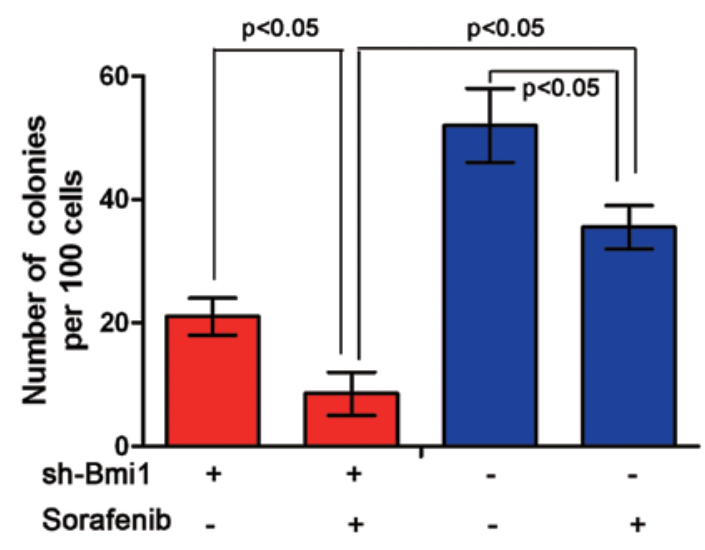

D

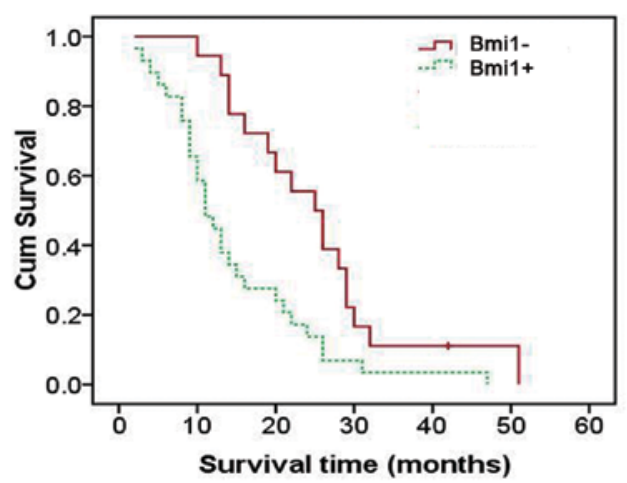

Figure 5. Bmil knockdown enhances the sensitivity of human hepatocellular carcinoma (HCC) cells to sorafenib. (A) The transfected cells were treated with $5 \mu \mathrm{M}$ sorafenib, and a clone formation assay was performed. (B) Quantitative analysis of the clone formation assays. (C) The transfected cells were treated with $5 \mu \mathrm{M}$ sorafenib; $72 \mathrm{~h}$ later, a cell viability assay was performed. (D) Kaplan-Meier survival curves for HCC patients with regard to Bmil expression. 
derived from the HepG2-shControl cells $\left(1,492 \mathrm{~mm}^{3} / 1.24 \mathrm{~g}\right.$, Fig. 4A-C). Similar data were obtained from the MHCC97shControl and MHCC97-shBmi1 cells. Collectively, these data demonstrate that the knockdown of Bmil inhibits cell proliferation and tumorigenicity in vivo.

We then examined the expression of the Bmil and Ki67 proteins by immunohistochemistry in all the xenograft tumor tissues formed by the HepG2-shControl, HepG2-shBmi1, MHCC97-shControl and MHCC97-shBmil cells (Fig. 4D). The tumor tissues formed by HepG2-shBmil expressed lower levels of Ki67 and Bmil than those formed by the HepG2shControl cells (Fig. 4E). Similar results were obtained for the MHCC97-shControl and MHCC97-shBmil cells. These results demonstrate that Bmil promotes tumor formation and the development of HCC through accelerated cell proliferation.

Bmil knockdown enhances the sensitivity of human HCC cells to sorafenib. Sorafenib is currently the most promising molecular targeted drug for human HCC. To investigate whether Bmil affects the sensitivity of HCC cells to sorafenib, we treated HepG2-shBmi1 cells and HepG2-shControl cells with sorafenib at various concentrations. In this figure, the results display the effects of sorafenib at a concentration of $5 \mu \mathrm{M}$. As shown in Fig. 5A and B, the number of colonies formed by the HepG2-shBmil cells was lower than that formed by the HepG2-shControl cells. In addition to forming the least number of colonies, the HepG2-shBmi1 cells, with or without sorafenib, all showed a significantly lower proliferation rate than the control cells throughout the experimental period, as measured by cell viability assay (MTT assay) $(\mathrm{P}<0.05$; Fig. 5C). We also observed that patients $(n=47)$ who showed negative Bmil expression had longer survival times, as revealed by a Kaplan-Meier analysis ( $\mathrm{P}<0.05$; Fig. 5D).

\section{Discussion}

Bmil is widely expressed in a variety of human tumors, including medulloblastomas (24), non-small cell lung (25), breast (26), prostate (27) and bladder cancer (28). Microarray analyses of multiple types of cancer have also indicated that Bmil is a predictor of metastasis and poor survival (29). In this study, we examined the expression of Bmil in HCC, non-tumor liver tissues and normal liver tissues, and observed that Bmi1 was overexpressed in HCC. To determine the role of Bmil in the growth of HCC, we established stable Bmil-knockdown cells in the HepG2 and MHCC97 cell lines by inducing the expression of an shRNA that targeted Bmil-specific mRNA in Balb/c-nude mice. We found that the knockdown of Bmi1 expression inhibited cell growth and colony formation by inducing cell cycle arrest in the G0/G1 phase. We also observed that the knockdown of Bmil expression attenuated the development and growth of the implanted HCC cells. These observations demonsrtate that Bmil promotes $\mathrm{HCC}$ cell tumor formation by accelerating cell growth. Our results are in agreement with those from previous reports showing that Bmil knockdown inhibits cell growth and reduces metastasis in various types of cancer $(17,30,31)$.

Ample evidence exists demonstrating the correlation between Bmil expression and the self-renewal and pluripotency maintenance of both normal and CSCs $(18,32,33)$. Bmi1 has been reported to be highly expressed in $\mathrm{CD}_{133^{+}}$murine liver CSCs and to play a role in the maintenance of hepatic stem progenitor cells (18). This finding is of great interest as the knockdown of Bmil expression decreases the tumorsphere formation ability under the sphere culture condition that allows the proliferation of only CSCs and progenitor cells. Previous studies have shown that Bmil controls self-renewal and the cell cycle by regulating the tumor suppressor proteins, p16INK4a and p14ARF, in cells $(34,35)$. In the present study, we found that Bmil knockdown induced cell cycle arrest at the cellular level. Therefore, Bmil may regulate the growth of HCC by promoting the proliferation of both CSCs and cancer cells. Furthermore, it would be of interest to examine the reliable surface markers of HCC CSCs and to investigate the role of Bmil in the proliferation and differentiation of HCC CSCs.

To date, sorafenib is the first and only drug that has been shown to be beneficial for the overall survival of patients with HCC. Preclinical studies have shown that sorafenib potently decreases HCC proliferation. Animal studies as well as clinical trials have shown that the co-administration of therapeutic agents should be more beneficial than monotherapies $(36,37)$. Experiments in vivo and in vitro have clearly established that Bmil is an oncogene that plays critical roles in promoting CSC self-renewal and tumorigenesis in HCC. We also assessed the sensitivity of HepG2-shBmil and MHCC97-shBmil cells to sorafenib in terms of their proliferation. We demonstrated that sorafenib had a more potent inhibitory effect on shBmil cell proliferation than that of the shControl cells. These results demonstrate that reduced Bmil protein levels inhibit hepatocarcinogenesis by targeting CSCs and providing a potential therapeutic target for the future treatment of HCC.

In conclusion, the results from our study showed that Bmil was overexpressed in HCC compared with the adjacent non-tumor tissues, as indicated by western blot analysis and immunohistochemical staining. The knockdown of Bmil in the HCC cell lines inhibited cell growth and colony formation by arresting the cell cycle in the G0/G1 phase. Additionally, tumorsphere formation, representing in vitro CSC selfrenewal, was also repressed. Furthermore, Bmil knockdown also enhanced the sensitivity of HCC cells to the chemotherapeutic agent sorafenib. These results support the idea that the suppression of Bim1 expression significantly inhibits hepatocarcinogenesis.

\section{References}

1. Parkin DM, Bray F, Ferlay $\mathrm{J}$ and Pisani P: Estimating the world cancer burden: Globocan 2000. Int J Cancer 94: 153-156, 2001.

2. Bertolini G, Roz L, Perego P, et al: Highly tumorigenic lung cancer $\mathrm{CD} 133^{+}$cells display stem-like features and are spared by cisplatin treatment. Proc Natl Acad Sci USA 106: 16281-16286, 2009.

3. Bonnet D and Dick JE: Human acute myeloid leukemia is organized as a hierarchy that originates from a primitive hematopoietic cell. Nat Med 3: 730-737, 1997.

4. Uchida N, Buck DW, He D, et al: Direct isolation of human central nervous system stem cells. Proc Natl Acad Sci USA 97: 14720-14725, 2000.

5. Al-Hajj M, Wicha MS, Benito-Hernandez A, Morrison SJ and Clarke MF: Prospective identification of tumorigenic breast cancer cells. Proc Natl Acad Sci USA 100: 3983-3988, 2003.

6. Ricci-Vitiani L, Lombardi DG, Pilozzi E, et al: Identification and expansion of human colon-cancer-initiating cells. Nature 445: 111-115, 2007. 
7. Rountree CB, Ding W, He L and Stiles B: Expansion of CD133expressing liver cancer stem cells in liver-specific phosphatase and tensin homolog deleted on chromosome 10-deleted mice. Stem Cells 27: 290-299, 2009.

8. Van der Lugt NM, Domen J, Linders K, et al: Posterior transformation, neurological abnormalities, and severe hematopoietic defects in mice with a targeted deletion of the bmi-1 protooncogene. Genes Dev 8: 757-769, 1994.

9. Pirrotta V: Polycombing the genome: PcG, trxG, and chromatin silencing. Cell 93: 333-336, 1998.

10. Kranc KR, Bamforth SD, Braganca J, Norbury C, van Lohuizen M and Bhattacharya S: Transcriptional coactivator Cited2 induces Bmil and Mel18 and controls fibroblast proliferation via Ink4a/ ARF. Mol Cell Biol 23: 7658-7666, 2003.

11. Saito M, Handa K, Kiyono T, et al: Immortalization of cementoblast progenitor cells with Bmi-1 and TERT. J Bone Miner Res 20: 50-57, 2005.

12. Kim JH, Yoon SY, Kim CN, et al: The Bmi-1 oncoprotein is overexpressed in human colorectal cancer and correlates with the reduced $\mathrm{p} 16 \mathrm{INK} 4 \mathrm{a} / \mathrm{p} 14 \mathrm{ARF}$ proteins. Cancer Lett 203: 217-224, 2004

13. Sasaki M, Ikeda H, Itatsu K, et al: The overexpression of polycomb group proteins Bmil and EZH2 is associated with the progression and aggressive biological behavior of hepatocellular carcinoma. Lab Invest 88: 873-882, 2008.

14. Vrzalikova K, Skarda J, Ehrmann J, et al: Prognostic value of Bmi-1 oncoprotein expression in NSCLC patients: a tissue microarray study. J Cancer Res Clin Oncol 134: 1037-1042, 2008.

15. Park IK, Qian D, Kiel M, et al: Bmi-1 is required for maintenance of adult self-renewing haematopoietic stem cells. Nature 423: 302-305, 2003.

16. Lessard J and Sauvageau G: Bmi-1 determines the proliferative capacity of normal and leukaemic stem cells. Nature 423: 255-260, 2003

17. Liu S, Dontu G, Mantle ID, et al: Hedgehog signaling and Bmi-1 regulate self-renewal of normal and malignant human mammary stem cells. Cancer Res 66: 6063-6071, 2006.

18. Chiba T, Seki A, Aoki R, et al: Bmil promotes hepatic stem cell expansion and tumorigenicity in both Ink4a/Arf-dependent and -independent manners in mice. Hepatology 52: 1111-1123, 2010.

19. Glinsky GV: Stem cell origin of death-from-cancer phenotypes of human prostate and breast cancers. Stem Cell Rev 3: 79-93, 2007.

20. Wang H, Pan K, Zhang HK, et al: Increased polycomb-group oncogene Bmi-1 expression correlates with poor prognosis in hepatocellular carcinoma. J Cancer Res Clin Oncol 134: 535-541, 2008.

21. Zhang Y, Li B, Ji ZZ and Zheng PS: Notch1 regulates the growth of human colon cancers. Cancer 116: 5207-5218, 2010.

22. Tomuleasa C, Soritau O, Rus-Ciuca D, et al: Isolation and characterization of hepatic cancer cells with stem-like properties from hepatocellular carcinoma. J Gastrointestin Liver Dis 19: 61-67, 2010 .
23. Zhu Z, Hao X, Yan MX, et al: Cancer stem/progenitor cells are highly enriched in CD133+CD44+ population in hepatocellular carcinoma. Int J Cancer 126: 2067-2078, 2010.

24. Leung C, Lingbeek M, Shakhova O, et al: Bmil is essential for cerebellar development and is overexpressed in human medulloblastomas. Nature 428: 337-341, 2004.

25. Vonlanthen S, Heighway J, Altermatt HJ, et al: The bmi-1 oncoprotein is differentially expressed in non-small cell lung cancer and correlates with INK4A-ARF locus expression. Br J Cancer 84: 1372-1376, 2001

26. Kim JH, Yoon SY, Jeong SH, et al: Overexpression of Bmi-1 oncoprotein correlates with axillary lymph node metastases in invasive ductal breast cancer. Breast 13: 383-388, 2004.

27. Berezovska OP, Glinskii AB, Yang Z, Li XM, Hoffman RM and Glinsky GV: Essential role for activation of the Polycomb group (PcG) protein chromatin silencing pathway in metastatic prostate cancer. Cell Cycle 5: 1886-1901, 2006.

28. Qin ZK, Yang JA, Ye YL, et al: Expression of Bmi-1 is a prognostic marker in bladder cancer. BMC Cancer 9: 61, 2009.

29. Glinsky GV, Berezovska O and Glinskii AB: Microarray analysis identifies a death-from-cancer signature predicting therapy failure in patients with multiple types of cancer. J Clin Invest 115: 1503-1521, 2005.

30. Chiba T, Miyagi S, Saraya A, et al: The polycomb gene product BMI1 contributes to the maintenance of tumor-initiating side population cells in hepatocellular carcinoma. Cancer Res 68: 7742-7749, 2008.

31. Cui H, Hu B, Li T, et al: Bmi-1 is essential for the tumorigenicity of neuroblastoma cells. Am J Pathol 170: 1370-1378, 2007.

32. Bruggeman SW, Hulsman D, Tanger E, et al: Bmil controls tumor development in an Ink4a/Arf-independent manner in a mouse model for glioma. Cancer Cell 12: 328-341, 2007.

33. Yu CC, Lo WL, Chen YW, et al: Bmi-1 regulates Snail expression and promotes metastasis ability in head and neck squamous cancer-derived ALDH1 positive cells. J Oncol 2011: pii: 609259, 2011.

34. Park IK, Morrison SJ and Clarke MF: Bmi1, stem cells, and senescence regulation. J Clin Invest 113: 175-179, 2004.

35. Dimri GP, Martinez JL, Jacobs JJ, et al: The Bmi-1 oncogene induces telomerase activity and immortalizes human mammary epithelial cells. Cancer Res 62: 4736-4745, 2002.

36. Jane EP, Premkumar DR and Pollack IF: Coadministration of sorafenib with rottlerin potently inhibits cell proliferation and migration in human malignant glioma cells. J Pharmacol Exp Ther 319: 1070-1080, 2006.

37. Yu C, Friday BB, Lai JP, et al: Cytotoxic synergy between the multikinase inhibitor sorafenib and the proteasome inhibitor bortezomib in vitro: induction of apoptosis through Akt and c-Jun NH2-terminal kinase pathways. Mol Cancer Ther 5: 2378-2387, 2006. 